RUNNING HEAD: Mind wandering distorts interval timing

\title{
Time contracts and temporal precision declines when the mind wanders
}

Devin B. Terhune, ${ }^{1,2}$ Madeleine Croucher, ${ }^{1}$ David Marcusson-Clavertz, ${ }^{3,4}$ \& James S. P. Macdonald ${ }^{5}$

${ }^{1}$ Department of Experimental Psychology, University of Oxford, Oxford, UK

${ }^{2}$ Department of Psychology, Goldsmiths, University of London, London, UK

${ }^{3}$ Department of Psychology, Lund University, Lund, Sweden

${ }^{4}$ Department of Biobehavioral Health, Pennsylvania State University, State College, PA, USA

${ }^{5}$ Department of Psychology, University of Roehampton, London, UK

\section{Corresponding author:}

Devin B. Terhune

Department of Psychology

Goldsmiths, University of London

New Cross, London SE14 6NW

Tel: $+44(0) 2070785148$

Fax: +44 (0) 1865310447

Email: d.terhune@gold.ac.uk

Word count: 4,000 


\section{Abstract}

Our perception of time varies considerably from moment to moment but how this variability relates to endogenous fluctuations in attentional states remains poorly understood. Here we tested the hypothesis that perceptual decoupling during mind wandering would distort interval timing. In two studies with different visual interval timing paradigms, we found that mind wandering states were characterized by underestimation of intervals and a decline in temporal discrimination. Further analyses suggested that temporal contraction during mind wandering, but not a decline in temporal discrimination, could be attributed in part to attentional lapses. These results highlight the role of transient fluctuations in attentional states in intra-individual variability in time perception and have implications for the behavioral markers, and costs and benefits, of mind wandering.

Keywords: attention; interval timing; metacognition; mind wandering; oddball; predictive coding

\section{Public significance statement}

The way we experience time changes from one moment to the next: we may underestimate or overestimate time or we may perceive it as constant or fragmented. Previous research has identified factors that influence our perception of time but the variables that underlie spontaneous fluctuations in time perception have been neglected. Here we examined whether changes in our tendency to mind wander (to think about things other than the task at hand) might explain some of the variability in our time perception. In two studies, we show that participants underestimated time and were poorer at discriminating different temporal intervals when they were mind wandering. Temporal contraction seems to be attributable, in part, to lapses in our attention whereas poorer temporal discrimination may be due to impaired perception when mind wandering. These results help to clarify the role of spontaneous changes in attentional states to variations in our perception of time. 
Our ability to accurately perceive time plays an instrumental role in a wide range of psychological functions including speech processing, motor control, and our moment-to-moment conscious experience (Allman, Teki, Griffiths, \& Meck, 2014; Matthews \& Meck, 2016; Wittmann, 2013). Time perception in the milliseconds to seconds range (interval timing) is known to exhibit considerable intra-individual differences but the source of this variability is poorly understood (Matthews \& Meck, 2014; Terhune, Sullivan, \& Simola, 2016). Identifying the factors underlying this variability will help to advance contemporary models of interval timing (Coull, Cheng, \& Meck, 2011; Gu, van Rijn, \& Meck, 2015; Merchant, Harrington, \& Meck, 2013; Yin, Terhune, Smythies, \& Meck, 2016) and strengthen our understanding of heterogeneity in cognition and perception more broadly (Matthews \& Meck, 2016).

One as of yet unexplored possibility is that moment-to-moment interval timing varies with transient fluctuations in attentional states. The average person spends as much as a third of their waking life engaging in thought that is unrelated to the current activity (Kane et al., 2007; Kane et al., in press; Marcusson-Clavertz, Cardeña, \& Terhune, 2016), a phenomenon known as mind wandering (Schooler et al., 2011; Smallwood \& Schooler, 2015). Evidence suggests that during mind wandering, one's attention is coupled to task-independent thoughts and concomitantly dissociated from perceptual input, resulting in an attenuation of sensory processing (perceptual decoupling; (Barron, Riby, Greer, \& Smallwood, 2011; Smallwood, Beach, Schooler, \& Handy, 2008). The magnitude of processing resources directed to a timed interval is related to its perceived duration such that greater, or lesser, allocation of resources is associated with temporal dilation, or contraction, respectively (Berry, Li, Lin, \& Lustig, 2014; Buhusi \& Meck, 2009). The association between perceived duration and resource allocation suggests that perceptual decoupling during mind wandering states will lead to distortions in interval timing.

\section{Study 1}

This study examined how mind wandering impacts interval timing. Mind wandering was measured through self-report by the inclusion of attention probes on a trial-by-trial basis (Smallwood \& Schooler, 2009, 2015). Previous research has consistently validated the use of such probes: performance is reliably 
impaired on trials preceding self-reported mind wandering episodes relative to those in which task-focus was maintained (Smallwood \& Schooler, 2009). Participants completed an oddball task involving a homogeneous train of standard stimuli of fixed duration punctuated by infrequent (oddball) stimuli of varying duration. This task has similarities with the Sustained Attention to Response Task (Robertson, Manly, Andrade, Baddeley, \& Yiend, 1997), a go-no-go task with infrequent no-go trials, which is the most widely used behavioral index of mind wandering (Smallwood \& Schooler, 2015). Oddball stimuli are typically perceived as dilated relative to standards plausibly because the repetition of standards gives rise to predictive coding (Eagleman \& Pariyadath, 2009; Pariyadath \& Eagleman, 2007; Schindel, Rowlands, \& Arnold, 2011). This has been proposed to result in the perceived contraction of standards due to repetition suppression and the concomitant dilation of oddballs due to the violation of sensory predictions (Grill-Spector, Henson, \& Martin, 2006; Pariyadath \& Eagleman, 2007; Wiggs \& Martin, 1998). There is evidence that subsecond timing is subserved in part by distributed sensory-specific mechanisms (Bueti, 2011) and thus we expected that perceptual decoupling during mind wandering would suppress the extent to which oddballs violate sensory predictions, resulting in their perceived contraction (rather than customary dilation) and a decline in temporal discrimination.

\section{Methods}

Thirty-four right-handed individuals $\left(M_{\mathrm{Age}}=25, S E=0.7 ; 56 \%\right.$ female $)$ consented to participate in this study in accordance with local ethical approval.

Participants completed an oddball task in which infrequent stimuli (green circles) of varying duration $(325 \mathrm{~ms}, 375,425,475,525,575,625,675)$ were randomly embedded $\left(8^{\text {th }}, 9^{\text {th }}\right.$, or $10^{\text {th }}$ position $)$ within a train of 11 homogeneous standard stimuli (blue circles) of constant duration $(500 \mathrm{~ms})$ with inter-stimulus intervals of $200 \mathrm{~ms}$. Participants judged with their right hand whether the oddball was shorter or longer in duration than the standards and whether they were "on-task" (task-focus) or "off-task" (mind wandering) during the stimulus train using a Cedrus ${ }^{\circledR}$ response pad (Cedrus Corporation, San Pedro, CA). Fingerresponse mappings and stimulus colours were counterbalanced across participants. Stimuli were presented 
at a distance of $70 \mathrm{~cm}$, subtending a visual angle of $2.45^{\circ 2}$, with E-Prime ${ }^{\circledR}$ (2.0, Psychology Software Tools, Pittsburgh, PA). Participants completed one 8-trial practice block followed by five 96-trial blocks.

Data were modeled using the Palamedes toolbox (Kingdom \& Prins, 2010; Prins \& Kingdom, 2009) for MATLAB ${ }^{\circledR}$ (2011a, MathWorks, Natick, MA). The proportions of long responses ( $p($ long)) at each interval for task-focus and mind wandering states were independently fitted with logistic functions defined by four parameters: threshold $\alpha$, slope $\beta$, guess rate $\gamma$, and lapse rate $\lambda$. We fixed $\gamma$ at 0 because of the 2-alternative forced-choice response format and $\lambda$ at 0.1 to allow for occasional lapses (Kingdom \& Prins, 2010). $\alpha$ and $\beta$ were set as free parameters and estimated using maximum likelihood estimation. In order to determine whether the effects of mind wandering on performance were driven by attentional lapses, we repeated the analyses with $\lambda$ as a free parameter. The duration corresponding to the $50 \%$ threshold on the psychometric function was used as the point of subjective equality (PSE), which corresponds to the oddball duration that is perceived as equivalent to the standards (values less than and greater than $500 \mathrm{~ms}$ reflect relative overestimation and underestimation, respectively). Temporal precision was computed with the Weber fraction (WF), which is the difference limen $[(t(p($ long $)=0.75)$ $t(p($ long $)=0.25)) / 2$, where $t$ is the stimulus duration at the respective response proportion location on the fitted psychometric function], divided by the PSE, with lower values reflecting superior precision. Eight participants' data were omitted because of poor model fit in one or both states (three in task-focus, seven in mind wandering; $p$ Devs $<.05$; (Kingdom \& Prins, 2010)); these data are presented in Fig. 1b. The greater proportion of omitted cases in mind wandering states can be attributed to an insufficient number of such reports. To further corroborate analyses of WFs, we computed error rates and $d^{\prime}$, a signal detection theory measure of discrimination ability (Green \& Swets, 1966).

The data violated assumptions of parametric statistical tests and were analyzed using Bootstrap resampling. Each statistic was computed on resampled data (10,000 samples) and the $95 \%$ confidence intervals (CIs; (bias-corrected and accelerated method; Efron, 1987) of these distributions are reported. We report both uncorrected Spearman correlations and those in which outliers have been removed 
(adjusted boxplot rule; Carling, 2000; Pernet, Wilcox, \& Rousselet, 2012); in all cases the two coefficients exhibit convergent statistical significance.

\section{Results}

Perceived duration

Participants reported mind wandering in $12 \%[9,23]$ of trials. They marginally overestimated the duration of oddballs during task-focus states, $M d n$ PSE=488ms [462, 500], (Fig. 1a), but experienced significant contraction of oddballs during mind wandering, $M d n=567 \mathrm{~ms}[524,703]$. The majority of participants displayed a larger PSE $[85 \% ; 65,96]$, reflecting greater underestimation, during mind wandering than task-focus states (Fig. 1c). Bootstrap resampling revealed a Mdn $\Delta$ PSE (MW-TF) of 105ms [75, 213], indicating that participants underestimated oddballs when mind wandering, $d=0.32[0.21,0.82]$. This shift was unrelated to mind wandering rate, $r_{\mathrm{s}}=.30[-.13, .63]$ (no outliers).

\section{Temporal discrimination}

WF values were lower during task-focus, $M d n=.12[.09, .17]$, than mind wandering, $M d n=.23[.21, .56]$, states (Fig. 1d), suggesting a decline in temporal precision during the latter. The majority of participants exhibited this effect $[92 \% ; 77,100]$, reflecting a significant $\Delta \mathrm{WF}$ of $.15[.09, .33], d=0.43[0.22,0.92]$. Further analyses revealed superior performance in task-focus than mind wandering states for error rates (task-focus: .20 [.15, .25]; mind wandering: .37 [.32, .42]; $M d n \Delta: .14[.11, .16], d=1.48$ [1.01, 2.18]), and $d^{\prime}$ values (task-focus: 1.82 [1.50, 2.15]; mind wandering: .79 [.37, 1.05]; $M d n \Delta: .97[.78,1.30], d=1.53$ $[1.06,2.64])$. Mind wandering rate was unrelated to mind wandering-specific shifts in WFs, $r_{\mathrm{s}}=.31[-.13$, $.66], r_{\mathrm{s}}($ outliers removed $)=.30[-.21, .68]$, error rates, $r_{\mathrm{s}}=-.04[-.44, .37], r_{\mathrm{s}}($ outliers removed $)=-.02[-.44$ $.43]$, or $d^{\prime}, r_{\mathrm{s}}=.10[-.36, .49]$ (no outliers). Both temporal contraction and reduced temporal precision during mind wandering are also present in the data of excluded participants (Fig. 1b). 

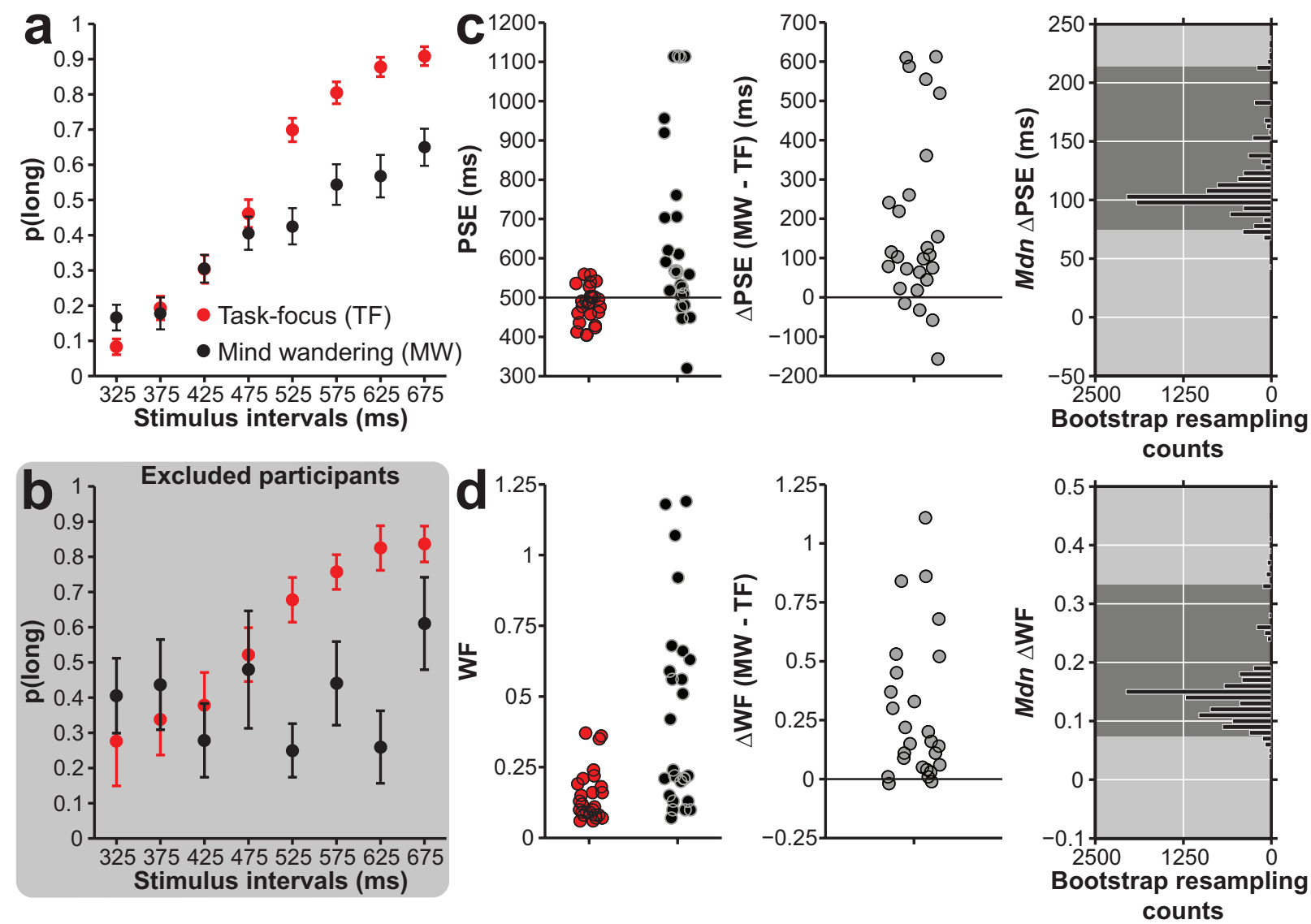

Figure 1. Oddball task performance parameters. (a) Proportion of long responses $[p$ (long)] as a function of oddball interval and attentional state. (b) Participants excluded due to poor model fit. (c) PSE (point of subjective equality) in attentional states (the solid line represents the duration of the standards; values larger than 500ms reflect underestimation of oddball intervals); $\triangle \mathrm{PSE}$ (MW-TF) (the solid line represents the null hypothesis of no difference); Bootstrap resampling counts of the $M d n \Delta \mathrm{PSE}$ (the dark gray area reflects the $95 \%$ confidence region of the distribution). (d) WF (Weber fraction) in attentional states.

\section{Interval timing with variable lapse rates}

The foregoing analyses assumed a fixed $\lambda$ so that timing-specific effects could be modeled as a function of self-reported mind wandering episodes. We next investigated whether the impact of mind wandering was attributable to attentional lapses by treating $\lambda$ as a free parameter. Estimated $\lambda$ values were lower in task-focus, $.02[.00, .06]$, than mind wandering, .03 $[.00, .30]$, states, although this difference did not achieve significance, $M d n \Delta \lambda<.001[-.01, .18], d=0.00$ [-0.08, 1.15]. PSEs remained lower in task-focus, 
$M d n=478 \mathrm{~ms}[450,492]$, than mind wandering, $M d n=543 \mathrm{~ms}[489,615]$, states, $M d n \Delta \mathrm{PSE}=66[7,138]$, $d=0.21[0.02,0.63]$. Similarly, WF values remained lower in task-focus, $M d n=.13[.09, .18]$, than mind wandering, $M d n=.27[.13, .59]$, states, $M d n \Delta \mathrm{WF}=.12[.05, .28], d=0.19[0.07,0.48]$.

\section{Discussion}

These results suggest that mind wandering states are characterized by temporal contraction and a decline in temporal discrimination. These effects are plausibly driven by perceptual decoupling during mind wandering. Perceptual decoupling may produce a decline in visual processing of to-be-timed stimuli leading to impaired temporal discrimination and may diminish the representational deviance of oddball stimuli, resulting in underestimation due to predictive coding (Eagleman \& Pariyadath, 2009; Pariyadath \& Eagleman, 2007; Schindel et al., 2011). The magnitude of both effects was smaller when attentional lapses were incorporated into the analyses although neither effect appears to be wholly driven by such lapses.

The frequency of mind wandering was lower than in research on its frequency in daily life (Kane et al., 2007; Kane et al., in press; Killingsworth \& Gilbert, 2010; Marcusson-Clavertz et al., 2016). This may be attributable to the high cognitive demand associated with the task (Marcusson-Clavertz et al., 2016; Rummel \& Boywitt, 2014), the high probe frequency (Seli, Carriere, Levene, \& Smilek, 2013; Smallwood \& Schooler, 2015), and the faster stimulus presentation rate (McKiernan, D'Angelo, Kaufman, \& Binder, 2006), all of which may have attenuated mind wandering by regularly reinforcing the task set. This high probe frequency was deemed necessary to ensure sufficient data were collected for the psychophysical analyses although future research may consider selectively presenting probes based on the cumulative mind wandering rate for each stimulus interval.

A further deviation from the extant literature is that the oddball effect in task-focused states $(\sim 2 \%$ dilation) was smaller than in previous studies ( 10-50\%; (Rose \& Summers, 1995; Seifried \& Ulrich, 2010; Tse, Intriligator, Rivest, \& Cavanagh, 2004; Ulrich, Nitschke, \& Rammsayer, 2006). This attenuation may have been caused by the need to monitor one's attentional state and the necessary 
redirection of resources from the task toward ensuring meta-awareness of attentional states. This may have similarly augmented temporal contraction during mind wandering. However, insofar as participants needed to monitor attention on a trial-by-trial basis irrespective of their attentional state, it is unlikely that mind wandering-specific distortions in timing are attributable to the need to monitor attention.

\section{Study 2}

The oddball paradigm used in Study 1 captures a specific illusion that is hypothesized to be driven by predictive coding (Eagleman \& Pariyadath, 2009; Pariyadath \& Eagleman, 2007; Schindel et al., 2011). However, the disruption of this illusion during mind wandering may not be representative of the impact of mind wandering on interval timing more generally. For this reason, this study examined whether distorted timing during mind wandering could be replicated in a widely-used paradigm requiring the constant timing of stimuli. Toward this end, participants completed a temporal bisection task in which they judged whether comparison intervals were closer in duration to previously learned short or long intervals (Kopec \& Brody, 2010). Motivated by research highlighting the importance of meta-awareness in mind wandering (Smallwood \& Schooler, 2015) and time perception (Lamotte, Izaute, \& Droit-Volet, 2012), we further explored whether mind wandering impacts metacognition (Fleming \& Lau, 2014; Maniscalco \& Lau, 2012) of interval timing.

\section{Methods}

Thirty-seven right-handed individuals $\left(M_{\mathrm{Age}}=23, S E=1 ; 52 \%\right.$ female) consented to participate in this study in accordance with local ethical approval. None of the participants had taken part in Study 1.

Participants completed a temporal bisection task in which they were first trained on two standard intervals $(360 \mathrm{~ms}$ and $640 \mathrm{~ms})$ and subsequently judged whether comparison intervals $(360 \mathrm{~ms}, 400,440$, $480,520,560,600,640)$ were closer in duration to the short or the long standards. Each trial consisted of a blank $1000 \mathrm{~ms}$ interstimulus interval followed by a comparison interval (a blue circle with the same dimensions as in Study 1). After a second 1000ms interstimulus interval, participants were presented with 
a 2-dimensional grid (Macdonald, Mathan, \& Yeung, 2011) upon which they judged 1) whether the comparison interval was closer to the short or the long standard interval; 2) their confidence in this judgment; and 3) the extent to which they were "on-task" (task-focus) or "off-task" (mind wandering) by pressing the left mouse button with their right index finger. Each dimension of the grid was labeled with two anchors ("Sure short" vs. "Sure long"; "On-task" vs. "Off-task") with both the position of the anchors and the dimensions counterbalanced across participants. Participants completed five practice trials (to familiarize themselves with the response grid) and 20 training trials with 10 presentations of each of the standards, followed by four blocks of 192 trials, amounting to 96 presentations of each interval.

Data were analyzed in the same way as in Study 1. The duration corresponding to the $50 \%$ threshold on the psychometric function was used as the bisection point (BP), which corresponds to the duration of the comparison interval that is perceived as equidistant from the two standards, with larger values reflecting relative temporal underestimation of comparison intervals. Six participants' data were omitted because of poor model fit in one condition ( 1 in task-focus states, 5 in mind wandering states) $(p$ devs $<.05)$ (Prins \& Kingdom, 2009); these data are presented in Fig. 2b. We also computed error rates and $d^{\prime}$ for each attentional state. To measure, metacognition of timing, we down-sampled pseudocontinuous confidence judgments (0 to 1$)$ to a five-point scale (i.e., $0-0.2: 1 ; 0.2-0.4: 2 ; 0.4-0.6: 3 ; 0.6-$ 0.8: $4 ; 0.8-1: 5)$ and submitted the data to receiver-operating-characteristic (ROC) analysis independently for task-focus and mind wandering state data using maximum likelihood estimation in order to compute meta- $d^{\prime}$, a measure of metacognitive sensitivity (Maniscalco \& Lau, 2012), which indexes the extent to which an individual's confidence judgments predict performance. Meta- $d^{\prime}$ is calculated in the same units $(S D \mathrm{~s})$ as $d^{\prime}$ and thus the two can be contrasted using meta- $d^{\prime}-d^{\prime}$ (metacognitive efficiency (Rounis, Maniscalco, Rothwell, Passingham, \& Lau, 2010), which quantifies the optimality of one's metacognitive sensitivity, namely how close it is to discrimination performance, with negative values reflecting poorer sensitivity (Fleming \& Lau, 2014). 


\section{Results}

Perceived duration

Participants reported mind wandering in $25 \%[20,31]$ of trials and displayed a tendency to underestimate comparison intervals during mind wandering states (Fig. 2a). BPs were similar in task-focus, $M d n=494 \mathrm{~ms}$ [471, 505], and mind wandering, $M d n=491 \mathrm{~ms}$ [476, 524], states, although bootstrap resampling revealed a $M d n \Delta \mathrm{BP}$ (MW-TF) of $16 \mathrm{~ms}[6,45]$ (Fig. 2c), suggesting that participants underestimated comparison intervals when mind wandering, $d=0.22[0.07,0.60]$, with $71 \%[52,84]$ of participants exhibiting this effect.

\section{Temporal discrimination}

Participants exhibited lower WFs, reflecting superior temporal precision, during task-focus, $M d n=.16$ $[.13, .20]$, than during mind wandering, $M d n=.22[.18, .26]$, states, representing a significant shift, $M d n$ $\Delta \mathrm{WF}=.04[.02, .07], d=0.42[0.13,0.86]$ (Fig. 2d). The majority of participants $[81 \% ; 65,94]$ exhibited this effect. Participants also displayed lower error rates during task-focus, $M d n=.28$ [.23, .31], than mind wandering, $M d n=.34[.31, .37]$, states, $M d n \Delta: .06[.03, .07], d=0.84[0.35,1.31]$, and higher $d^{\prime}$ during task-focus, $M d n=1.18[0.96,1.52]$, than mind wandering, $M d n=0.87[0.75,1.12]$, states, $M d n \Delta: 0.29$ [.14, $.37], d=0.73[0.30,1.17]$. Temporal contraction and reduced temporal precision during mind wandering are also apparent in the data of excluded participants (Fig. 2b).

\section{Metacognition of interval timing}

Metacognition of interval timing did not differ significantly across attentional states. Specifically, there were no differences in metacognitive sensitivity (meta- $d^{\prime}$; task-focus: $1.00[0.82,1.22]$; mind wandering: $0.66[0.57,1.10] ; M d n \Delta: 0.18[-.09, .37], d=0.42[-0.23,0.87])$, or efficiency (meta- $d^{\prime}-d^{\prime}$; task-focus: -.26 $[-.35,-.14]$; mind wandering: $-.10[-.21, .05]), \operatorname{Mdn} \Delta:-.12[-.27, .09], d=0.30[-0.25,0.74]$. 

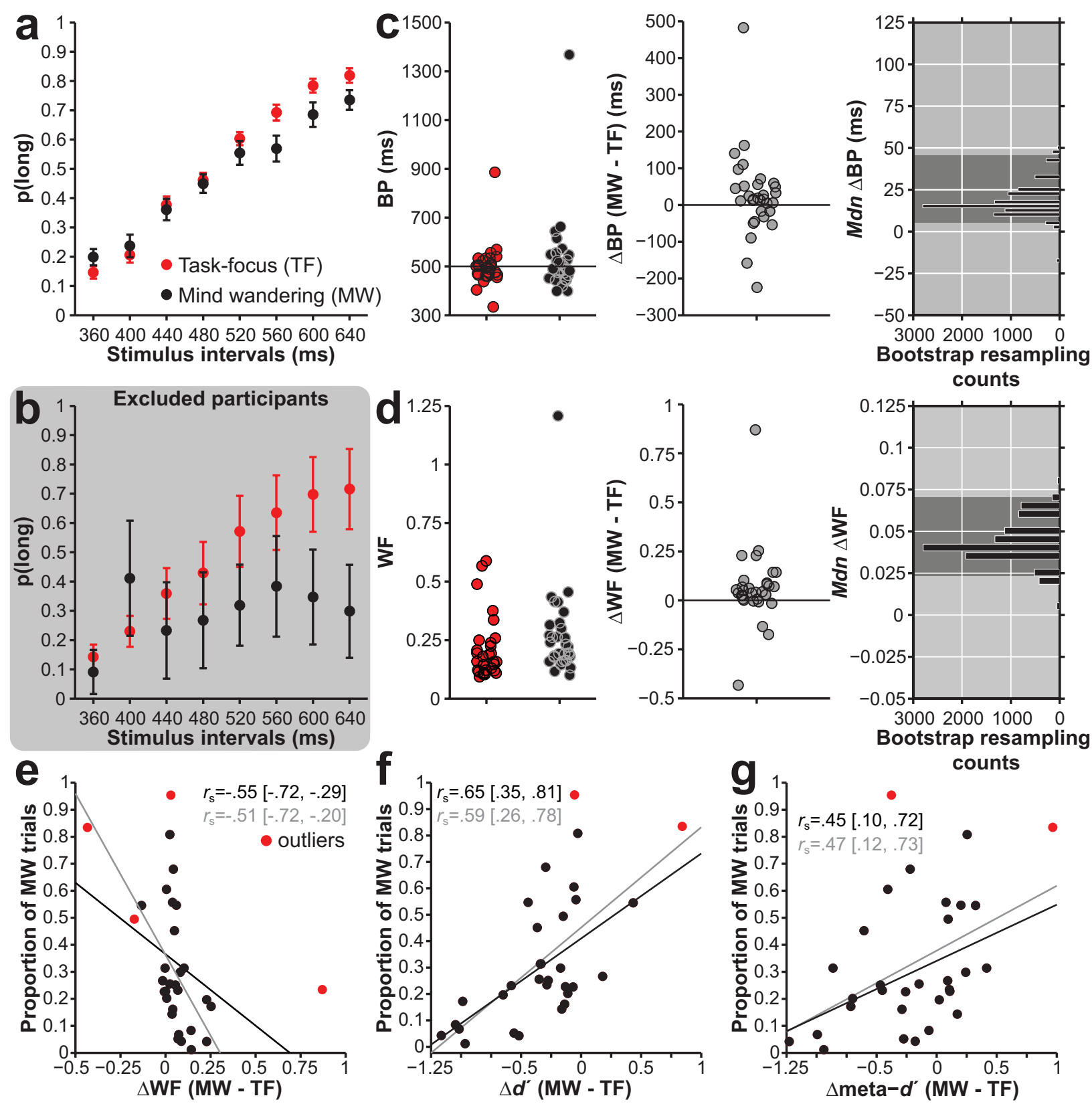

Figure 2. Temporal bisection task performance parameters. (a) Proportion of long responses $[p$ (long)] as a function of comparison interval and attentional state. (b) Excluded participants (due to poor model fit). (c) BP (bisection point) in attentional states (the solid line represents the objective BP; values larger than $500 \mathrm{~ms}$ reflect underestimation of comparison intervals); $\triangle \mathrm{BP}(\mathrm{MW}-\mathrm{TF}$ ) (the solid line represents the null hypothesis of no difference); Bootstrap resampling counts of the Mdn $\Delta \mathrm{BP}$ (the dark gray area reflects the $95 \%$ confidence region of the distribution). (d) WF (Weber fraction) in attentional states. (e-g) Correlations between the proportion of trials with self-reported mind wandering and the $\Delta$ in (e) WF, (f) $d^{\prime}$, and (g) metacognitive sensitivity (meta- $d^{\prime}$ ). Red markers reflect outliers (see Method). Reported values are uncorrected Spearman correlations on all data (black) and skipped Spearman correlations after the omission of outliers (gray). Bracketed values reflect bootstrap 95\% CIs. Regression lines for all data (black) and data after outlier omission (gray) are included for reference. 
Interval timing as a function of mind wandering frequency

Mind wandering rate differentially correlated with shifts in different performance parameters during mind wandering states. Although mind wandering rate was unrelated to $\Delta \mathrm{BP}, r_{\mathrm{s}}=.09[-.29, .46] ; r_{\mathrm{s}}$ (outliers removed $)=.05[-.35, .46]$, higher mind wandering rates were associated with smaller declines in WF (Fig. 2e), error rates, $r_{\mathrm{s}}=-.58[-.79,-.23], r_{\mathrm{s}}($ outliers removed $)=-.58[-.80,-.20]$, and $d^{\prime}$ values (Fig. 2f) during mind wandering states. Similarly, mind wandering rate was associated with smaller declines in metacognitive sensitivity (meta- $\left.d^{\prime}\right)$ (Fig. 2g), but not metacognitive efficiency (meta- $\left.d^{\prime}-d^{\prime}\right), r_{\mathrm{s}}=.30[-.03$, $.55], r_{\mathrm{s}}($ outliers removed $)=.24[-.12, .55]$, during mind wandering states.

Interval timing with variable lapse rates

As in Study 1, we examined whether the observed effects were attributable to attentional lapses. Estimated $\lambda$ values were higher in mind wandering, $.11[.05, .22]$, than task-focus, $.06[.02, .13]$, states, $\operatorname{Mdn} \Delta: .01[.00, .09]$, although this effect was very small, $d=0.06[0.00,0.71]$. BPs no longer differed between task-focus, $M d n=474 \mathrm{~ms}[460,496]$, and mind wandering, $M d n=459 \mathrm{~ms}[442,491]$, states, $M d n$ $\Delta$ BP: $-6 \mathrm{~ms}[-42,20], d=0.02[-0.16,0.31]$, although WF values remained lower in task-focus, $M d n=.18$ $[.12, .21]$, than mind wandering, $M d n=.25[.18, .29]$, states, $M d n \Delta \mathrm{WF}: .05[.02, .07], d=0.48[0.18,0.81]$. These results suggest that temporal contraction, but not reduced temporal precision, during mind wandering is driven by attentional lapses.

\section{Discussion}

This study replicated the principal results of Study 1: participants tended to underestimate temporal intervals, and display poorer temporal precision, when mind wandering. The magnitude of temporal contraction was slightly smaller than in Study 1 (representing $\sim 20 \%$ and $\sim 30 \%$ shifts of a $S D$, respectively) whereas declines in precision were comparable in the two studies ( $40 \%$ shift of a $S D)$. The precision results were further corroborated by analyses of discrimination ability. 
The results of this study partially departed from those of Study 1 when we considered whether lapse rates could account for the impact of mind wandering on interval timing. When lapse rates were free to vary, temporal contraction was no longer observed during mind wandering states whereas the decline in temporal precision remained stable. By contrast, in Study 1, both effects were attenuated, but not completely disrupted. This discrepancy may simply be due to the larger temporal contraction effect in Study 1 , which is plausibly attributable to additive effects of predictive coding and attentional lapses, whereas the corresponding effect in Study 2 may be solely due to the latter. Two further potential contributing factors were the smaller mind wandering rate in Study $1(12 \%)$ relative to Study $2(25 \%)$ and the absence of differential lapse rates across attentional states in the former. These effects may have allowed lapses to exert a greater impact on perceived duration in Study 2.

A notable set of results is that participants who mind wandered more frequently exhibited smaller declines in temporal discrimination and metacognitive sensitivity during mind wandering states, suggesting that frequent mind wandering is associated with reduced proneness to its deleterious effects. These associations are potentially driven by variability in meta-awareness (see, e.g., Smallwood, McSpadden, \& Schooler, 2007): individuals with superior meta-awareness of their attentional states may exhibit broadly superior cognitive functioning (cf. (Levinson, Smallwood, \& Davidson, 2012)) and thus be less susceptible to the detrimental effects of mind wandering. Alternatively, the results may relate to variability in the adjudication criteria by which participants judged their attentional states. Some participants may only report mind wandering during pronounced episodes, which might be expected to be associated with elevated perceptual distortions. Nevertheless, insofar as these effects were not observed in Study 1 , they should be interpreted with caution.

\section{General Discussion}

In two studies we demonstrate that mind wandering is characterized by distorted interval timing. During mind wandering episodes, participants underestimated subsecond intervals and exhibited a decline in temporal precision, but no change in metacognition of timing. These results complement research 
demonstrating that interval timing varies as a function of the allocation of processing resources to a stimulus (Buhusi \& Meck, 2009; Gu et al., 2015; Matthews \& Meck, 2016) and specifically highlights the influence of transient changes in attention on momentary shifts in interval timing (Berry et al., 2014; Matthews \& Meck, 2014). Fluctuations in attentional states may be driven by variations in occipital cortex excitability (Macdonald et al., 2011), which is consistent with research showing that temporal underestimation of visual intervals is associated with elevated local cortical inhibition in primary visual cortex (Terhune, Russo, Near, Stagg, \& Cohen Kadosh, 2014). Further research will benefit from contrasting these effects with other factors contributing to intra-individual variability in perceived duration, such as fluctuations in striatal dopamine receptor availability (Soares, Atallah, \& Paton, 2016; Terhune et al., 2016).

The principal aim of this study was to test the hypothesis that perceptual decoupling during mind wandering disrupts interval timing. Our results are only partially consistent with this hypothesis as they point to potentially distinct mechanisms by which mind wandering impacts timing. The results suggest that attentional lapses during mind wandering states underlie a moderate to substantial proportion of the influence of mind wandering on perceived duration. By contrast, lapses do not seem to substantially impact temporal discrimination. Declines in temporal discrimination during mind wandering are perhaps better explained by perceptual decoupling, whereby attention is redirected from sensory input toward task-unrelated mental representations, resulting in impaired perception (Barron et al., 2011; Schooler et al., 2011; Smallwood et al., 2008). These differential effects are consistent with evidence that estimated lapse rates in psychophysical models are closely linked to attentional states rather than sensory mechanisms (Kingdom \& Prins, 2010). Further indirect evidence for this dissociation is provided by the correlations with mind wandering rates: the latter were related to mind wandering-specific declines in temporal discrimination, but not to temporal contraction.

An open question is the temporal locus of the impact of attentional lapses on interval timing. One possibility is that lapses alter perceived duration by delaying the onset of stimulus timing: participants miss the onset of the target and thereby perceive the stimulus as contracted. However, a delayed onset of 
timing would have produced a rightward shift of psychometric functions, including greater accuracy for short intervals, but that was not observed. Alternatively, the temporal loci of attentional lapses may be more diffuse and produce periodic interruptions in temporal attention at various stages of stimulus processing. These explanations may be distinguished by measuring the perceived frequency of a second stimulus dimension (e.g., colour) that varies over time (Coull, Hwang, Leyton, \& Dagher, 2012; Coull, Vidal, Nazarian, \& Macar, 2004). If variability in the alternation of stimulus colour is experimentally weighted toward one colour at the onset of the stimulus, a delayed onset explanation would predict a larger judgment bias toward the other colour whereas a periodic lapse explanation would not.

Distorted interval timing may account for a common marker of mind wandering. One of the most widely observed behavioral correlates of mind wandering is an increase in response time variability (Esterman, Noonan, Rosenberg, \& Degutis, 2013; McVay \& Kane, 2009; Seli, Cheyne, \& Smilek, 2013). This increase is plausibly due in part to a decline in temporal precision during tasks requiring responses after a certain fixed amount of time has passed. One salient feature of our results is that distorted interval timing during mind wandering states appears to be either specific to, or at least more pronounced for, the timing of longer intervals. It is possible that short intervals may more readily capture attention and thus are processed in a similar way across task-focus and mind wandering states.

These results also have consequences for the costs and benefits of mind wandering (Smallwood \& Schooler, 2015). Mind wandering during tasks requiring precise timing of the environment, such as operating a motor vehicle (Yanko \& Spalek, 2014), will deleteriously impact performance. In contrast, mind wandering during repetitive tasks (Avni-Babad \& Ritov, 2003) or painful episodes (Kam, Xu, \& Handy, 2014) may help them pass more quickly, thereby representing a specific valuable function of temporal contraction.

\section{Acknowledgements}

This study was supported by a Marie Sklowdowska-Curie Intra-European Fellowship within the $7^{\text {th }}$ European Community Framework Programme to D.B.T. 


\section{References}

Allman, M. J., Teki, S., Griffiths, T. D., \& Meck, W. H. (2014). Properties of the internal clock: Firstand second-order principles of subjective time. Annual Review of Psychology, 65, 743-771. doi:10.1146/annurev-psych-010213-115117

Avni-Babad, D., \& Ritov, I. (2003). Routine and the perception of time. Journal of Experimental Psychology: General, 132(4), 543-550. doi:10.1037/0096-3445.132.4.543

Barron, E., Riby, L. M., Greer, J., \& Smallwood, J. (2011). Absorbed in thought: The effect of mind wandering on the processing of relevant and irrelevant events. Psychol Sci, 22(5), 596-601. doi:10.1177/0956797611404083

Berry, A. S., Li, X., Lin, Z., \& Lustig, C. (2014). Shared and distinct factors driving attention and temporal processing across modalities. Acta Psychologica, 147, 42-50. doi:10.1016/j.actpsy.2013.07.020

Bueti, D. (2011). The sensory representation of time. Frontiers in integrative neuroscience, 5, 34. doi:10.3389/fnint.2011.00034

Buhusi, C. V., \& Meck, W. H. (2009). Relative time sharing: New findings and an extension of the resource allocation model of temporal processing. Philosophical Transactions of the Royal Society of London. Series B: Biological Sciences, 364(1525), 1875-1885. doi:10.1098/rstb.2009.0022

Carling, K. (2000). Resistant outlier rules and the non-Gaussian case. Computational Statistics \& Data Analysis, 33, 249-258.

Coull, J. T., Cheng, R. K., \& Meck, W. H. (2011). Neuroanatomical and neurochemical substrates of timing. Neuropsychopharmacology, 36(1), 3-25. doi:10.1038/npp.2010.113

Coull, J. T., Hwang, H. J., Leyton, M., \& Dagher, A. (2012). Dopamine precursor depletion impairs timing in healthy volunteers by attenuating activity in putamen and supplementary motor area. Journal of Neuroscience, 32(47), 16704-16715. doi:10.1523/JNEUROSCI.1258-12.2012 
Coull, J. T., Vidal, F., Nazarian, B., \& Macar, F. (2004). Functional anatomy of the attentional modulation of time estimation. Science, 303(5663), 1506-1508. doi:10.1126/science.1091573

Eagleman, D. M., \& Pariyadath, V. (2009). Is subjective duration a signature of coding efficiency? Philosophical Transactions of the Royal Society of London. Series B: Biological Sciences, 364(1525), 1841-1851. doi:10.1098/rstb.2009.0026

Efron, B. (1987). Better bootstrap confidence intervals. Journal of the American Statistical Association, 82(397), 171-185. doi:Doi 10.2307/2289144

Esterman, M., Noonan, S. K., Rosenberg, M., \& Degutis, J. (2013). In the zone or zoning out? Tracking behavioral and neural fluctuations during sustained attention. Cerebral Cortex, 23(11), 27122723. doi:10.1093/cercor/bhs261

Fleming, S. M., \& Lau, H. C. (2014). How to measure metacognition. Front Hum Neurosci, 8, 443. doi:10.3389/fnhum.2014.00443

Green, D., \& Swets, J. (1966). Signal detection theory and psychophysics. New York, NY: Wiley.

Grill-Spector, K., Henson, R., \& Martin, A. (2006). Repetition and the brain: Neural models of stimulusspecific effects. Trends Cogn Sci, 10(1), 14-23. doi:10.1016/j.tics.2005.11.006

Gu, B., van Rijn, H., \& Meck, W. H. (2015). Oscillatory multiplexing of neural population codes for interval timing and working memory. Neuroscience and Biobehavioral Reviews, 48C, 160-185. doi:10.1016/j.neubiorev.2014.10.008

Kam, J. W., Xu, J., \& Handy, T. C. (2014). I don't feel your pain (as much): The desensitizing effect of mind wandering on the perception of others' discomfort. Cogn Affect Behav Neurosci, 14(1), 286296. doi:10.3758/s13415-013-0197-z

Kane, M. J., Brown, L. H., McVay, J. C., Silvia, P. J., Myin-Germeys, I., \& Kwapil, T. R. (2007). For whom the mind wanders, and when: An experience-sampling study of working memory and executive control in daily life. Psychol Sci, 18(7), 614-621. doi:10.1111/j.14679280.2007.01948.x 
Kane, M. J., Gross, G. M., Chun, C. A., Smeekens, B. A., Meier, M. E., Silvia, P. J., \& Kwapil, T. R. (in press). For whom the mind wanders, and when, varies across laboratory and daily-life settings Psychological Science.

Killingsworth, M. A., \& Gilbert, D. T. (2010). A wandering mind is an unhappy mind. Science, 330(6006), 932. doi:10.1126/science.1192439

Kingdom, F. A. A., \& Prins, N. (2010). Psychophysics: A practical introduction. London, UK: Elsevier.

Kopec, C. D., \& Brody, C. D. (2010). Human performance on the temporal bisection task. Brain and Cognition, 74(3), 262-272. doi:10.1016/j.bandc.2010.08.006

Lamotte, M., Izaute, M., \& Droit-Volet, S. (2012). Awareness of time distortions and its relation with time judgment: A metacognitive approach. Conscious Cogn, 21(2), 835-842. doi:10.1016/j.concog.2012.02.012

Levinson, D. B., Smallwood, J., \& Davidson, R. J. (2012). The persistence of thought: Evidence for a role of working memory in the maintenance of task-unrelated thinking. Psychol Sci, 23(4), 375-380. doi:10.1177/0956797611431465

Macdonald, J. S., Mathan, S., \& Yeung, N. (2011). Trial-by-trial variations in subjective attentional state are reflected in ongoing prestimulus EEG alpha oscillations. Frontiers in psychology, 2, 82. doi:10.3389/fpsyg.2011.00082

Maniscalco, B., \& Lau, H. (2012). A signal detection theoretic approach for estimating metacognitive sensitivity from confidence ratings. Conscious Cogn, 21(1), 422-430. doi:10.1016/j.concog.2011.09.021

Marcusson-Clavertz, D., Cardeña, E., \& Terhune, D. B. (2016). Daydreaming style moderates the relation between working memory and mind wandering: Integrating two hypotheses. Journal of Experimental Psychology. Learning, Memory, and Cognition, 42(3), 451-464. doi: $10.1037 / x \operatorname{lm} 0000180$

Matthews, W. J., \& Meck, W. H. (2014). Time perception: The bad news and the good. Wiley Interdiscip Rev Cogn Sci, 5(4), 429-446. doi:10.1002/wcs.1298 
Matthews, W. J., \& Meck, W. H. (2016). Temporal cognition: Connecting subjective time to perception, attention, and memory. Psychological Bulletin, 142(8), 865-907. doi:10.1037/bul0000045

McKiernan, K. A., D'Angelo, B. R., Kaufman, J. N., \& Binder, J. R. (2006). Interrupting the "stream of consciousness": An fMRI investigation. Neuroimage, 29(4), 1185-1191. doi:10.1016/j.neuroimage.2005.09.030

McVay, J. C., \& Kane, M. J. (2009). Conducting the train of thought: Working memory capacity, goal neglect, and mind wandering in an executive-control task. Journal of Experimental Psychology. Learning, Memory, and Cognition, 35(1), 196-204. doi:10.1037/a0014104

Merchant, H., Harrington, D. L., \& Meck, W. H. (2013). Neural basis of the perception and estimation of time. Annual Review of Neuroscience, 36, 313-336. doi:10.1146/annurev-neuro-062012-170349

Pariyadath, V., \& Eagleman, D. (2007). The effect of predictability on subjective duration. PloS one, 2(11), e1264. doi:10.1371/journal.pone.0001264

Pernet, C. R., Wilcox, R., \& Rousselet, G. A. (2012). Robust correlation analyses: False positive and power validation using a new open source matlab toolbox. Frontiers in psychology, 3, 606. doi:10.3389/fpsyg.2012.00606

Prins, N., \& Kingdom, F. A. (2009). Palamedes: Matlab routines for analyzing psychophysical data. Available at http://www.palamedestoolbox.org/.

Robertson, I. H., Manly, T., Andrade, J., Baddeley, B. T., \& Yiend, J. (1997). 'Oops!': Performance correlates of everyday attentional failures in traumatic brain injured and normal subjects. Neuropsychologia, 35(6), 747-758.

Rose, D., \& Summers, J. (1995). Duration illusions in a train of visual stimuli. Perception, 24(10), 11771187.

Rounis, E., Maniscalco, B., Rothwell, J. C., Passingham, R. E., \& Lau, H. (2010). Theta-burst transcranial magnetic stimulation to the prefrontal cortex impairs metacognitive visual awareness. Cogn Neurosci, 1(3), 165-175. doi:10.1080/17588921003632529 
Rummel, J., \& Boywitt, C. D. (2014). Controlling the stream of thought: Working memory capacity predicts adjustment of mind-wandering to situational demands. Psychon Bull Rev, 21(5), 13091315. doi:10.3758/s13423-013-0580-3

Schindel, R., Rowlands, J., \& Arnold, D. H. (2011). The oddball effect: Perceived duration and predictive coding. $J$ Vis, 11(2). doi:10.1167/11.2.17

Schooler, J. W., Smallwood, J., Christoff, K., Handy, T. C., Reichle, E. D., \& Sayette, M. A. (2011). Meta-awareness, perceptual decoupling and the wandering mind. Trends Cogn Sci, 15(7), 319326. doi:10.1016/j.tics.2011.05.006

Seifried, T., \& Ulrich, R. (2010). Does the asymmetry effect inflate the temporal expansion of odd stimuli? Psychological Research, 74(1), 90-98. doi:10.1007/s00426-008-0187-x

Seli, P., Carriere, J. S., Levene, M., \& Smilek, D. (2013). How few and far between? Examining the effects of probe rate on self-reported mind wandering. Frontiers in psychology, 4, 430. doi:10.3389/fpsyg.2013.00430

Seli, P., Cheyne, J. A., \& Smilek, D. (2013). Wandering minds and wavering rhythms: Linking mind wandering and behavioral variability. Journal of Experimental Psychology: Human Perception and Performance, 39(1), 1-5. doi:10.1037/a0030954

Smallwood, J., Beach, E., Schooler, J. W., \& Handy, T. C. (2008). Going AWOL in the brain: Mind wandering reduces cortical analysis of external events. Journal of Cognitive Neuroscience, 20(3), 458-469. doi:10.1162/jocn.2008.20037

Smallwood, J., McSpadden, M., \& Schooler, J. W. (2007). The lights are on but no one's home: Metaawareness and the decoupling of attention when the mind wanders. Psychon Bull Rev, 14(3), 527533.

Smallwood, J., \& Schooler, J. W. (2009). Mind-wandering. In T. Bayne, A. Cleeremans, \& P. Wilken (Eds.), The Oxford companion to consciousness (pp. 443-445). Oxford, UK: Oxford University Press. 
Smallwood, J., \& Schooler, J. W. (2015). The science of mind wandering: Empirically navigating the stream of consciousness. Annual Review of Psychology, 66, 487-518. doi:10.1146/annurev-psych010814-015331

Soares, S., Atallah, B. V., \& Paton, J. J. (2016). Midbrain dopamine neurons control judgment of time. Science, 354(6317), 1273-1277. doi:10.1126/science.aah5234

Terhune, D. B., Russo, S., Near, J., Stagg, C. J., \& Cohen Kadosh, R. (2014). GABA predicts time perception. Journal of Neuroscience, 34(12), 4364-4370. doi:10.1523/JNEUROSCI.397213.2014

Terhune, D. B., Sullivan, J., \& Simola, J. (2016). Time dilates after spontaneous blinking. Current Biology, 26, R459-R460. doi:10.1016/j.cub.2016.04.010

Tse, P. U., Intriligator, J., Rivest, J., \& Cavanagh, P. (2004). Attention and the subjective expansion of time. Perception and Psychophysics, 66(7), 1171-1189.

Ulrich, R., Nitschke, J., \& Rammsayer, T. (2006). Perceived duration of expected and unexpected stimuli. Psychological Research, 70(2), 77-87. doi:10.1007/s00426-004-0195-4

Wiggs, C. L., \& Martin, A. (1998). Properties and mechanisms of perceptual priming. Current Opinion in Neurobiology, 8(2), 227-233.

Wittmann, M. (2013). The inner sense of time: How the brain creates a representation of duration. Nature Reviews Neuroscience, 14(3), 217-223. doi:10.1038/nrn3452

Yanko, M. R., \& Spalek, T. M. (2014). Driving with the wandering mind: The effect that mind-wandering has on driving performance. Human Factors, 56(2), 260-269.

Yin, B., Terhune, D. B., Smythies, J., \& Meck, W. H. (2016). Claustrum, consciousness, and time perception. Current Opinion in Behavioural Sciences, 8, 258-267.

doi:10.1016/j.cobeha.2016.02.032 